\title{
Aortic root aortopathy in bicuspid aortic valve associated with high genetic risk
}

\author{
Mingjia Ma ${ }^{1}$, Zongzhe Li ${ }^{2,3}$, Mohamed Abdulkadir Mohamed ${ }^{1}$, Ligang Liu' ${ }^{1}$ and Xiang Wei ${ }^{*}$
}

\begin{abstract}
Background: The bicuspid aortic valve (BAV) is prone to ascending aortic dilatation (AAD) involving both the tubular segment and the aortic root. The genetic factor was proposed as one of the most important mechanisms for AAD. We hypothesized that the rare genetic variants mainly contribute to the pathogenesis of aortic roots in affected individuals.

Methods: The diameter of aortic root or ascending aorta $\geq 40 \mathrm{~mm}$ was counted as AAD. The targeted next-generation sequencing of $13 \mathrm{BAV}$-associated genes were performed on a continuous cohort of 96 unrelated BAV patients. The rare variants with allele frequency $<0.05 \%$ were selected and analyzed. Variants frequency was compared against the Exome aggregation consortium database. The pathogenicity of the genetic variants was evaluated according to the American College of Medical Genetics and Genomics guidelines.

Results: A total of 27 rare nonsynonymous coding variants involving 9 genes were identified in 25 individuals. The burden analysis revealed that variants in GATA5, GATA6, and NOTCH1 were significantly associated with BAV. Eighty percent of the pathogenic variants were detected in root group. The detection rate of rare variants was higher in root dilatation group (71.4\%) compared with normal aorta (29.0\%) and tubular dilatation groups (29.6\%) $(P=0.018)$. The rare variant was identified as the independent risk factor of root dilatation $[P=0.014$, hazard ratio $=23.9,95 \%$ confidence interval (1.9-302.9)].
\end{abstract}

Conclusions: Our results presented a broad genetic spectrum in BAV patients. The rare variants of BAV genes contribute the most to the root phenotype among BAV patients.

Keywords: Bicuspid aortic valve, Next generation sequencing, Aortopathy, Rare variant

\section{Background}

Bicuspid aortic valve (BAV) is a common congenital valvular defect, affecting about $0.5-1 \%$ of the general population $[1,2]$. Nearly half of the BAV affected patients would undergo medical or surgical management during their lifetime due to the BAV-related valvular and/or aortic complications (i.e. aortic stenosis, aortic insufficiency, aortic aneurysm, and aortic dissection et al.) [3]. Patients

\footnotetext{
*Correspondence: xiangwee_hust@163.com

${ }^{1}$ Division of Cardiothoracic and Vascular Surgery, Tongji Hospital, Tongji Medical College, Huazhong University of Science and Technology, 1095\# Jiefang Ave., Wuhan 430030, People's Republic of China

Full list of author information is available at the end of the article
}

with BAV had a high risk of development of the ascending aorta dilatation (to a size above $4.0 \mathrm{~cm}$ ) [4]. Of them, about $15 \%$ of patients have a dilated aortic root frequently with aortic insufficiency at a young age [5]. According to a large community cohort, the risk of aneurysm formation is significantly higher in BAV patients than the general population, and about a quarter of the patients with BAV may ultimately underwent aortic surgery after BAV diagnosis. The risk of acute aortic emergencies, most commonly aortic dissection, is eightfold in patients with BAV disease compared with the general population [6].

The underlying mechanisms of BAV-associated aortopathy are widely discussed. There are two potentially competing or more likely complementary perspectives 
of the cause of BAV aortopathy, i.e. the hemorheological reason and genetic factors. The former argues that the fused-leaflet of BAV alters the blood flow and increases the shear stress in the tubular ascending aorta [7]. From the genetic view, the genetic defect may increase the risk of BAV aortopathy [8]. However, which kind of influence would play a more important role is still controversial.

The aortic valve and ascending aorta share some common embryological origin [9]. It is reasonable to hypothesize that rare variants of BAV associated genes are likely to be risk factors predisposing to BAV aortopathy, especially in the aortic root. Human BAV presenting together with aortopathy has been proved to be associated with rare variants in NOTCH1, TGFBR2, FBN1, and SMAD6 et al. $[5,10]$.

However, our knowledges of the inherited components affecting the clinical phenotype of BAV aortopathy are still limited. It could be argued that different types of BAV aortopathy may have different rare variants spectrum. By targeted next generation sequencing (NGS) of genes associated with BAV, we aimed to investigate distribution of rare variants in a large well-phenotyped BAV cohort.

\section{Methods}

\section{Study population}

From January 2015 to December 2019, 130 unrelated patients with BAV were continuously recruited from the division of cardiothoracic and vascular surgery of Tongji hospital. Patient's phenotypes of BAV were detected by echocardiographic examination, and confirmed by the cardiac surgeon during the operation. The pattern of BAV, as displayed in Fig. 1, was recorded according to the phenotype category described by Sievers et al. [11]. The inclusion criteria were patients with BAV underwent cardiac surgery in our institution and agreed to participate in the trial. The exclusion criteria consisted of unidentifiable valve phenotype, complex congenital heart disease, and malignant tumors. Finally, a total of 96 surgical patients were included in this study. Informed consents were acquired from all the participants. The local research ethics committee approved the study.

The aortic diameter was measured at least at 2 levels: sinus of Valsalva and the widest level of the ascending aorta. According to the 2014 European Society of Cardiology guidelines, the aortic diameter stretching $40 \mathrm{~mm}$ or greater is considered as aortopathy [12]. For patient with suspected aortopathy, computed tomography angiography was further required to evaluate the geometrical morphology of the ascending aorta. The root type aortopathy was defined as the largest diameter located in the aortic sinus. The tubular type aortopathy was defined as the largest segment located in the ascending aorta. The aortopathy classification was illustrated in Fig. 2.

\section{Targeted NGS}

A custom panel including 13 BAV genes was designed using Ion AmpliSeq ${ }^{\mathrm{TM}}$ designer software. The gene

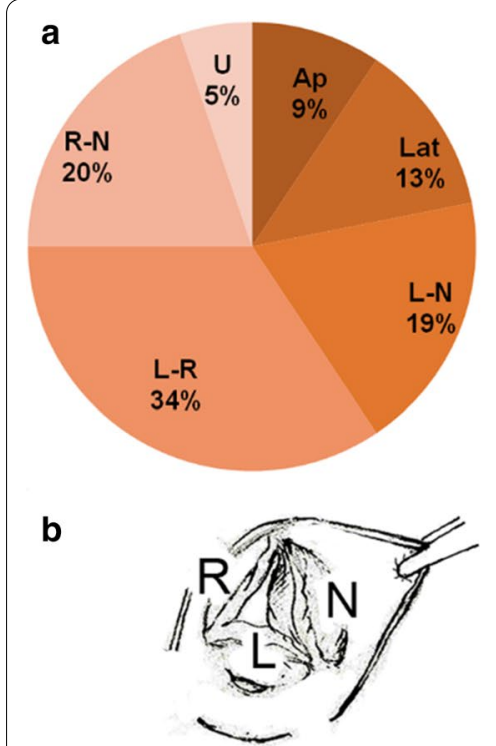

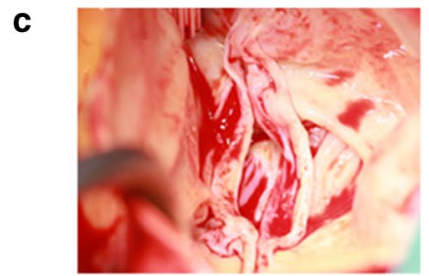

Ap

type 0

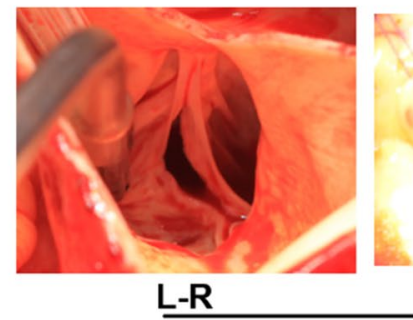

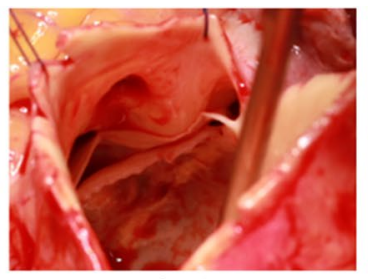

Lat

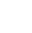

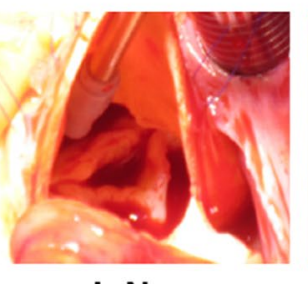

L-N

type 1

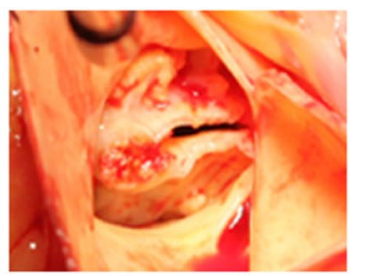

Unicuspid type 2

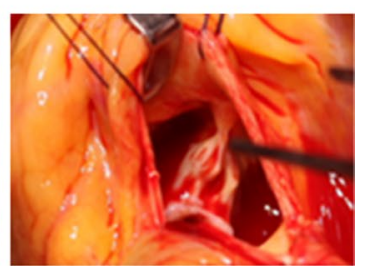

R-N

Fig. 1 The bicuspid aortic valve (BAV) classification. a composing rate of BAV in this study, b the aortic valve anatomic sketch (photographer's view), c the BAV types are confirmed during surgeries. Ap anterior-posterior, Lat lateral, L left coronary sinus, $N$ non-coronary sinus, $R$ right coronary sinus, $U$ unicuspid 

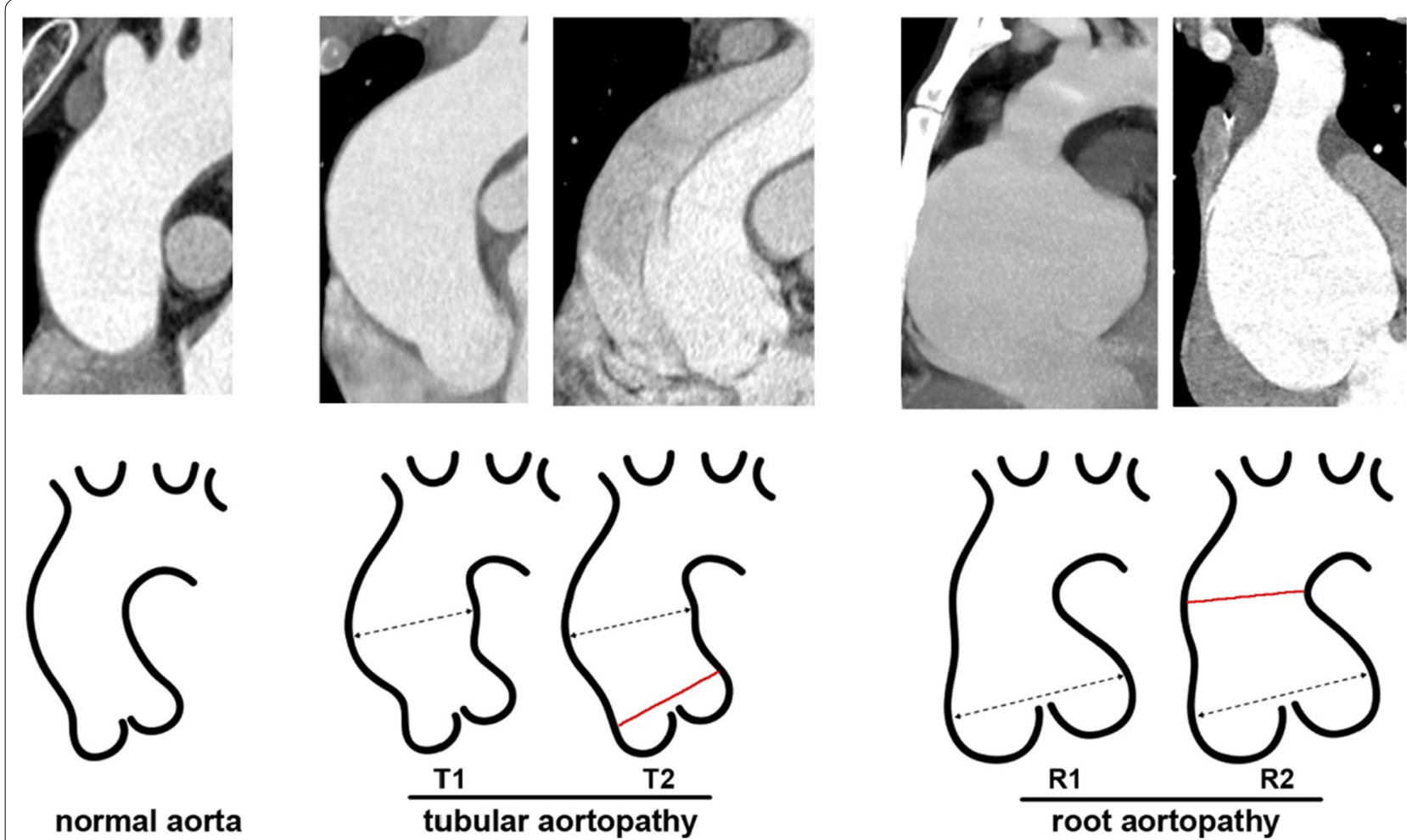

normal aorta

Fig. 2 Aortopathy classification. The dotted line marks the widest part of the aorta. The red line indicates the dilated segment. T1, ascending aorta dilatation with normal aortic root; T2, ascending aorta dilatation with aortic root expansion; R1, aortic root dilatation with normal ascending aorta; R2, aortic root dilatation with ascending aorta expansion

Table 1 Genes in the panel and rare variants burden comparison between patients and ExAC controls

\begin{tabular}{|c|c|c|c|c|}
\hline Gene & PMID & $\begin{array}{l}\text { Number of } \\
\text { variants in } 192 \\
\text { patients' alleles }\end{array}$ & $\begin{array}{l}\text { Allele count in } \\
\text { ExAC East Asia } \\
\text { group }\end{array}$ & $P$ value \\
\hline GATA6 & 29653232 & 3 & 19 in 7684 & 0.016 \\
\hline GATA5 & 24796370 & 2 & 7 in 7858 & 0.018 \\
\hline NOTCH1 & 16025100 & 9 & 130 in 7778 & 0.006 \\
\hline TGFBR1 & 25145529 & 1 & 11 in 8132 & 0.244 \\
\hline TGFBR2 & 23578328 & 2 & 31 in 8228 & 0.173 \\
\hline FBN1 & 24564502 & 5 & 132 in 8614 & 0.227 \\
\hline ELN & 14666267 & 2 & 68 in 8224 & 0.675 \\
\hline EGFR & 26708639 & 1 & 61 in 8592 & $>0.999$ \\
\hline FLNA & 21815255 & 2 & 122 in 6610 & $>0.999$ \\
\hline ACTA2 & 17994018 & 0 & 10 in 8432 & N.A \\
\hline GATA4 & 21330551 & 0 & 144 in 7658 & N.A \\
\hline$N K X 2-5$ & 25438918 & 0 & 10 in 7678 & N.A \\
\hline SMAD6 & 22275001 & 0 & 29 in 8050 & N.A \\
\hline
\end{tabular}

ExACThe Exome Aggregation Consortium, PMID PubMed unique identifier of reference, N.A. not available selecting criteria were causal genes of BAV and genes highly correlated to BAV aortopathy. The gene list and PubMed unique identifier of references were listed in Table 1. Genomic DNA was extracted from peripheral blood leucocytes using a blood DNA kit (Tiangen Biotech, Beijing, China). 96 samples were genetically evaluated using targeted resequencing on the Ion Proton platform (Life Technologies).

\section{Data analysis and filtering}

Sequencing data were processed with Ion Torrent Suite Software v5.0 (Life Technologies) to align reads to the human genome reference (hg19/GRCh37). The filtered variants with allele frequency $<0.05 \%$ in the online databases 1000 Genomes Project or the Exome aggregation consortium (ExAC) $[13,14]$ were selected as rare variants. We applied computational prediction algorithms: SIFT, Provean, PolyPhen-2 [15], and CADD [16] to predict the functional significance of the identified rare non-synonymous coding substitutions; Phylop scores to measure evolutionary conservation. Finally, we defined pathogenicity 
Table 2 Demographic and clinical characteristics of the bicuspid aortic valve

\begin{tabular}{|c|c|c|c|}
\hline Variables & No variant $(n=71)$ & Variant $(n=25)$ & $P$ value \\
\hline Age (years) & $45.6 \pm 12.7$ & $41.5 \pm 13.7$ & 0.173 \\
\hline Male (\%) & $58(81.7)$ & $16(64.0)$ & 0.070 \\
\hline BMI $\left(\mathrm{kg} / \mathrm{m}^{2}\right)$ & $22.0 \pm 3.4$ & $23.1 \pm 4.8$ & 0.242 \\
\hline Hypertension (\%) & $23(32.4)$ & $10(40.0)$ & 0.491 \\
\hline Diabetes mellites (\%) & $9(12.7)$ & $4(16.0)$ & 0.737 \\
\hline Bicuspid valve type (\%) & & 0.524 & \\
\hline Anterior-posterior & $8(11.3)$ & $1(4.0)$ & - \\
\hline Lateral & $8(11.3)$ & $4(16.0)$ & - \\
\hline L-N & $13(18.3)$ & $5(20.0)$ & - \\
\hline$L-R$ & $22(31.0)$ & $11(44.0)$ & - \\
\hline $\mathrm{R}-\mathrm{N}$ & $15(21.1)$ & $4(16.0)$ & - \\
\hline Unicuspid & $5(7.0)$ & 0 & - \\
\hline $\begin{array}{l}\text { Aortic valve disease } \\
(\%)\end{array}$ & & 0.983 & \\
\hline Aortic insufficiency & $27(38.0)$ & $10(40.0)$ & - \\
\hline Aortic stenosis & $18(25.4)$ & $6(24.0)$ & - \\
\hline Combined lesions & $26(36.6)$ & $9(36.0)$ & - \\
\hline $\begin{array}{l}\text { Infective endocarditis } \\
(\%)\end{array}$ & $13(18.3)$ & $4(16.0)$ & $>0.999$ \\
\hline Aortopathy (\%) & $23(32.4)$ & $11(44.0)$ & 0.297 \\
\hline Aortic dissection (\%) & $4(5.6)$ & $3(12.0)$ & 0.372 \\
\hline $\begin{array}{l}\text { Surgery for aortopa- } \\
\text { thy (\%) }\end{array}$ & $15(21.1)$ & $7(28.0)$ & 0.482 \\
\hline LVEF\% & $60.0 \pm 9.2$ & $58.6 \pm 10.8$ & 0.521 \\
\hline
\end{tabular}

$B M I$ body mass index, $L V E F$ left ventricular ejection fraction, $L$ left coronary sinus, $N$ non-coronary sinus, $R$ right coronary sinus, $S D$ standard deviation

of variants in accordance with the American College of Medical Genetics and Genomics (ACMG) guidelines [16].

The rare variants fund by NGS were then validated on an automated DNA sequencer ABI 3130 XL (Applied Biosystems, Foster City, CA) using the Big Dye Terminator v3.1 kit (Thermo Fisher Scientific, Waltham, MA, USA) following the manufacturer's guidelines.

\section{Copy number variants (CNVs)}

To detect potential large genomic structural variation such as deletions, duplications and other CNVs, the copy number of all sequenced regions was analyzed by amplicon coverage data.

\section{Rare variant burden analysis}

To assess the contribution of genetic variant risk to $\mathrm{BAV}$, the frequency of qualifying rare variants per gene in our patient's cohort were then compared to that in the ExAC East Asian database. The controlling variant was all nonsynonymous and loss-of-function variants with allele frequency $<0.05 \%$ among ExAC data base. The results of rare variant burden were list in Table 1.

\section{Statistical analysis}

Continuous variables were presented as mean \pm standard deviation, and compared using the t-test or the Mann-Whitney U-test. The one-way ANOVA test was employed for multigroup analysis. The post-hoc tests were processed in the pairwise comparisons manner. Categorical variables were expressed as percentages, and analyzed using the Fisher's exact test or the Chi-square test. The post-hoc test of $2 \times 3$ contingency table was performed with adjusted standardized residual analysis. The mosaic plot was employed to illustrated the relationship between aortic configuration and rare variants. The cell represented positive relationship between two variables was for blue shades. The multivariable logistic regress model was built for aortopathy risk factor analysis. All two-tailed $P<0.05$ was considered statistically significant. All statistical analyses were performed with $\mathrm{R}$ version 3.2.5 (Vienna, Austria).

\section{Results}

\section{The demographic characteristics}

The study enrolled 96 individuals. Of them, 74 males and 22 females, with a mean age of $44.6 \pm 13.0$ years (ranged from 15 to 67 years). The aortic dilatations were found in 34 patients (35.4\% of all the participants), of them, 22 patients underwent surgery for the aortic aneurysm or dissection. In this cohort, no aortic coarctation was observed. With regard to BAV type, left-right model is the most common type found in one-third of the BAV patients (34.4\%).

\section{Sequencing data}

The NGS was offered to all of the 96 participants. The average depth of coverage for the targeted regions was 1406 reads. The percentage of sequencing on target was $93.77 \%$. Greater than $50 \times$ coverage was obtained for $99.01 \%$ of the bases sequenced. A total of 27 rare nonsynonymous coding variants involving 9 genes were identified by targeted capture in $25 \mathrm{BAV}$ patients. Two patients harbored 2 rare variants, respectively. The variants comprised 24 missense, 1 frameshift, and 2 nonsense mutations. Of them, 14 rare variants had been reported in neither ExAC nor ClinVar database previously. The details of rare variants were presented in Additional file 1: Table S1.

The potential CNVs were validated by real-time polymerase chain reaction but no large deletion or duplication was detected. 

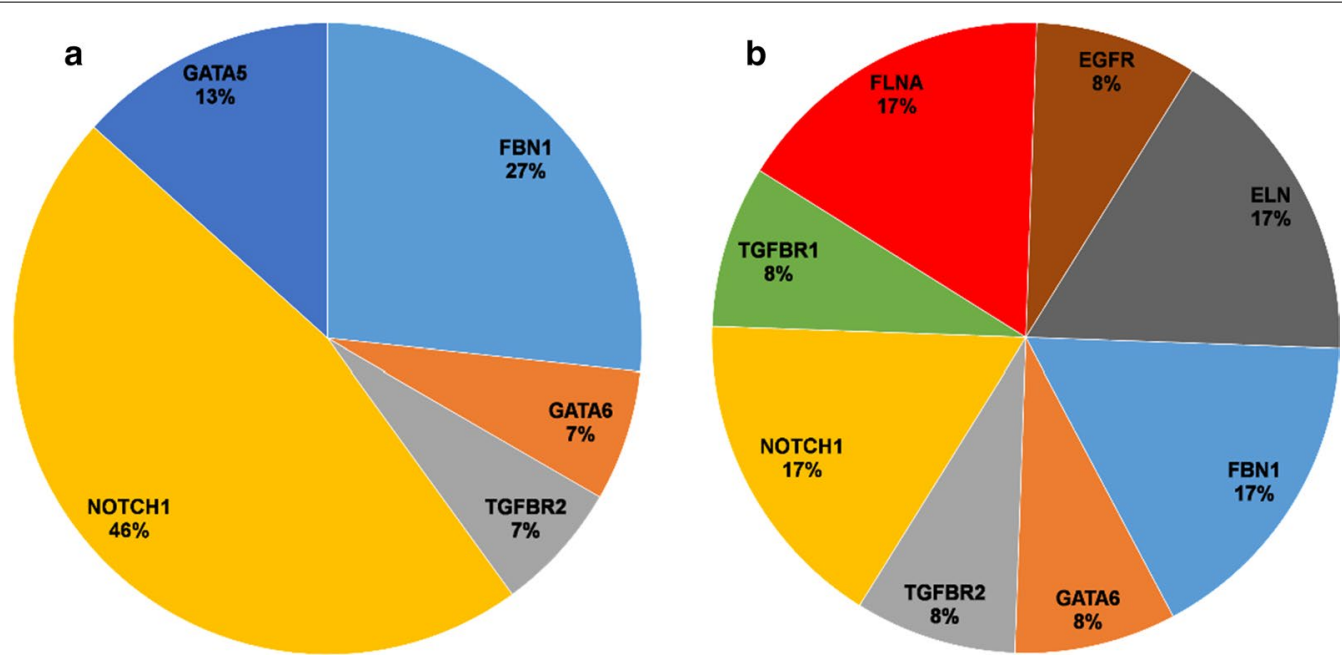

Fig. 3 The spectrum of rare variants detected in patients of normal aorta (a), and aortopathy (b)

\section{Rare variant burden analysis}

Of the 13 BAV genes, the frequency of rare nonsynonymous coding variants of genes GATA5, GATA6, and NOTCH1 were significant higher in the 96 BAV cohort compared to the ExAC online database.

\section{Genotype-phenotype correlation}

The patients were compared between rare variants group and no variants group. There was no significant difference of variables between the two groups as depicted in Table 2.

We then investigated the distribution of rare variants in the normal aorta group and aortopathy group (including both the root and tubular types). The variants number were comparable in two groups, i.e. 15 in normal group and 12 in aortopathy group, respectively. Interestingly, the constituent ratios of rare variants displayed distinct distribution characteristics between the two groups. In the normal aorta group, the frequency of NOTCH1 variant reached $46.7 \%$ of all detected rare variants. Oppositely, the variants in NOTCH1 accounts for $16.7 \%$ of all detected variants in aortopathy group, though the difference was not statistically significant $(P=0.217)$. Meanwhile, the number of involved genes in aortopathy group was more than that in the normal group (8: 5 genes). The rare variants distribution was illustrated in Fig. 3. This suggested that the BAV aortopathy may have a more complex genetic background than the normal group.

\section{BAV aortopathy}

One third of the BAV patients $(35.4 \%, 34 / 96)$ were diagnosed with aortopathy. We compared patients among normal aorta, tubular aortopathy, and root aortopathy groups. The patients of tubular aortopathy were the oldest, with a mean age of 53.6 years. In contrast, the aortic root aortopathy group was the youngest. The number

Table 3 Comparing aortopathy and normal ascending aorta

\begin{tabular}{|c|c|c|c|c|}
\hline Variables & Normal aorta $(n=62)$ & $\begin{array}{l}\text { Tubular aortopathy } \\
(\mathrm{n}=27)\end{array}$ & Root aortopathy $(n=7)$ & $\begin{array}{l}P \text { value } \\
\text { Total }\end{array}$ \\
\hline Onset age (years) & $41.7 \pm 12.7$ & $53.6 \pm 8.9 *$ & $35.0 \pm 11.8$ & $<0.001$ \\
\hline Male (\%) & $40(75.8)$ & $22(81.5)$ & $5(71.4)$ & 0.878 \\
\hline Diabetes mellitus (\%) & $6(9.7)$ & $5(18.5)$ & $2(28.6)$ & 0.102 \\
\hline Hypertension (\%) & $24(38.7)$ & $9(33.3)$ & 0 & 0.081 \\
\hline Variant burden (\%) & $14(22.6)$ & $6(22.2)$ & $5(71.4)^{+}$ & 0.018 \\
\hline Aortic dissection (\%) & $2(3.2)$ & $3(11.1)$ & $2(28.6)$ & 0.024 \\
\hline Infective endocarditis (\%) & $14(22.6)$ & $3(11.1)$ & 0 & 0.070 \\
\hline Multiple valves replacement (\%) & $15(24.2)$ & $1(3.7)$ & $1(14.3)$ & 0.070 \\
\hline
\end{tabular}

${ }^{*}$ With the ANOVA test for the onset age, statistically significant difference was found between normal aorta, tubular aortopathy and root aortopathy groups. Tubular group is the oldest according to the post-hoc test

${ }^{\dagger}$ For variant burden, Chi-square test indicated a statistically significant difference between three groups. Adjusted residual for root group was $2.3(p=0.00045)$, reaching the threefold Bonferroni-adjusted significance level of $p<0.0167)$ 


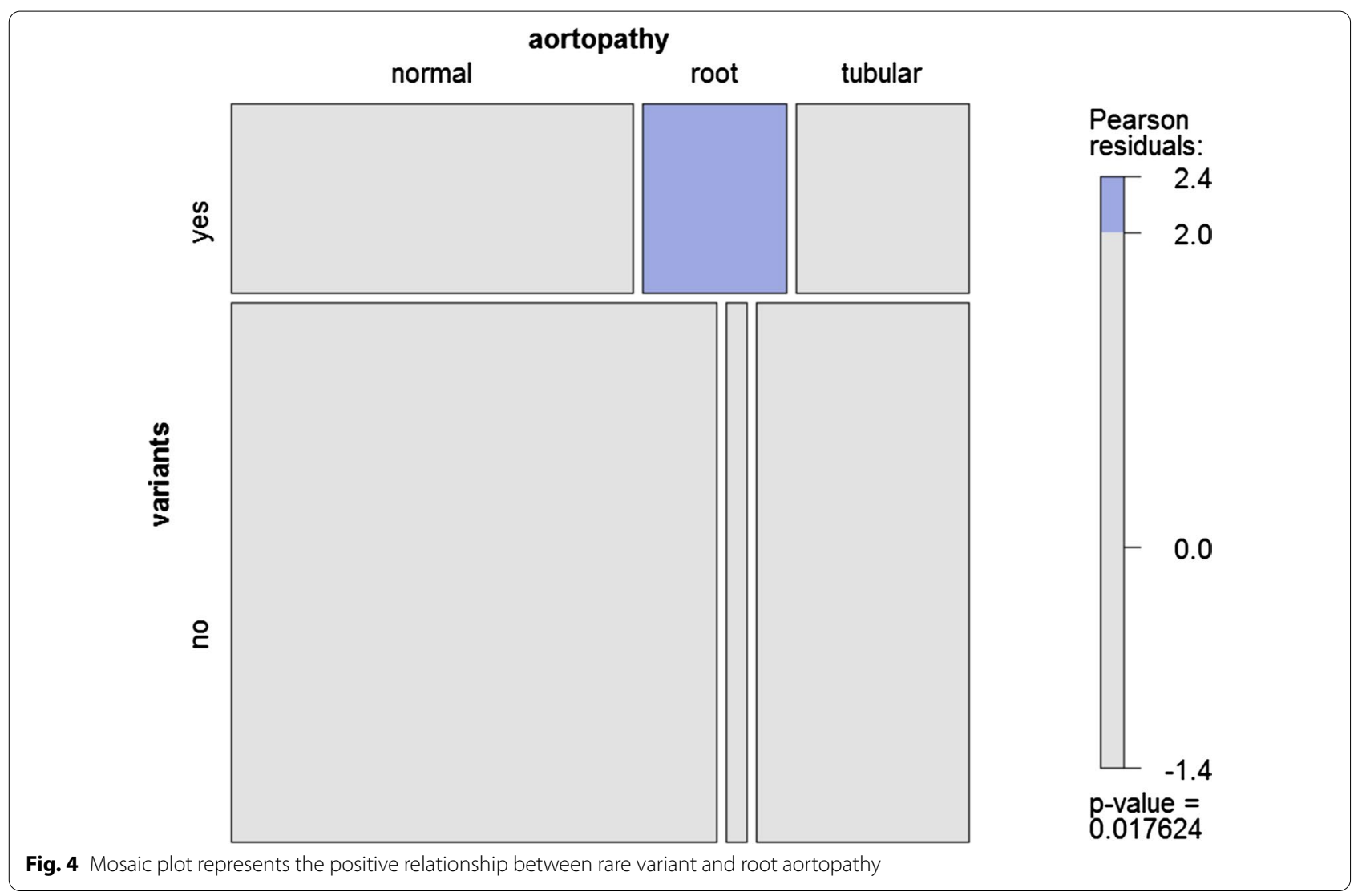

of tubular type cases was 4 times as high as root type (27:7). The aortic dissection was more often in root group $(28.6 \%, 2 / 7)$ than in normal $(3.2 \%, 2 / 62)$ and tubular $(11.1 \%, 3 / 27)$ groups $(P=0.048)$. Of note, none in root group had aortic calcification or infective endocarditis. The comparative results were presented in Table 3.

With regard to the genetic influence of the BAV aortopathy, there were $5(71.4 \%)$ patients in the root group harbored rare variants. Instead, number of rare variant case was $8(29.6 \%)$ in tubular group and $18(29.0 \%)$ in normal group, respectively. The patients of root type aortopathy had the highest frequency of rare variants among the BAV patients $(P=0.021)$. In the mosaic plot (Fig. 4$)$, the cell of rare variant and root aortopathy was for blue with the Pearson's residuals of 2.8, represented a positive relationship between the rare variant number and root aortopathy cases. In addition, a logistic-regress model was built for risk factors of root aortopathy, by including variables of rare variant, gender, age, body surface area, hypertension, aortic valve disease (insufficiency, stenosis, and combined lesions) and BAV type (type $0,1,2$ ). The rare variant was identified as the independent risk factor $[P=0.014$, hazard ratio $=23.9,95 \%$ confidence interval (1.9-302.9)].

\section{Discussion}

In the present study, we reported on targeted resequencing detection of the rare variants associated with BAV and related aortopathy. With a 13-gene panel, an overall rare variants detection rate of $26.0 \%$ in 96 patients was achieved. To the best of our knowledge, this study was the largest cohort by targeted resequencing accessing the pathogenic variants associated with sporadic BAV disease in the Eastern Asian population. According to our data, the BAV patients with aortopathy displayed more complex genetic heterogeneity than BAV patients with normal aorta. The root type aortopathy was strongly associated with the rare variants of BAV genes.

The flourishing availability of NGS technologies provided us the convenience to rapidly detect multiple variants in multiple genes, by which, a more comprehensive understanding of the heritability of the BAV and associated aortopathy would be reached. Bonachea et al. conducted a targeted sequencing assay of 78 unrelated BAV patients [17]. They identified 31 putative disease-causing variants in 16 individuals. Dargis et al. used a panel of 9 genes associated with BAV to study 48 patients [18], they identified 19 potentially pathogenic variants. Of them, 7 variants in NOTCH1 took the highest burden of deleterious variants. Some researchers also reported the NGS 
results of the BAV aortopathy. Girdauskas et al. used a 20 genes panel to investigate $63 \mathrm{BAV}$ patients with root aortopathy [19]. A total of 64 rare variants in 15 candidate genes were identified, of them 24 were potentially pathogenic/likely pathogenic variants. Gillis used a panel of 22 genes to study a large BAV/ thoracic aortic aneurysm cohort [10]. They identified 112 variants classified as pathogenic in 441 patients. In comparison with these previous studies, our cohort reaches a similar rare variants detection rate, suggests that the BAV might be a combination as a polygenic trait, many discrete genes being responsible for the disease.

Some studies have suggested that aortic dilatation of BAV is an inherent attribute of the disease. In a longterm follow-up research, the accelerated aortic dilatation in BAV was observed even independent of severe aortic stenosis or regurgitation and aortic coarctation [4]. Although the mechanism of aortic dilation has been long debated, the genetic theory was continuously advocated and investigated. Based on genome-wide single nucleotide polymorphism array, the early onset of thoracic aortic disease was demonstrated frequently in BAV patients, and associated with recurrent rare copy number variations [20]. These findings suggest that BAV and aortopathy might share some common molecular mechanism, the genetic defect may hurt the cardiac or vascular development, leading to the early onset of disease.

Several recent studies focused on the genic origin of BAV related aortopathy. Pepe et al. suggested FBN1 was an important cause of the aortic root dilatation in BAV [21]. Gillis et al. [10] considered variants in SMAD6 has a significant contribution to BAV aortopathy, with a variant burden of $2.5 \%$. In our study, there is a wide spectrum of rare genetic variants in patients with aortic dilatation, no gene plays an outstanding role.

NOTCH1 is the first gene proved to be associated with both familial and sporadic BAV cases. Altered Notch signaling causes malformations of the left ventricular outflow tract including BAV and facilitates the progress of aortic valve disease [22]. Controversy also exists in whether the NOTCH1 variant is a causal of BAV aortopathy. Girdauskas et al. found a wide spectrum of the variants in 19 of 63 patients with BAV root aortopathy, and NOTCH1 was the leading causal gene [19]. In contrast, Kent et. al investigated BAV related aortopathy by sequencing the NOTCH1 gene in $13 \mathrm{BAV}$ families. They failed to identified causal variant in the patients and concluded that the NOTCH1 variant contributed little to the noncalcified bicuspid aortic valve combined with ascending aortic aneurysm [23]. Ambitiously, in another multiple center sequencing study, NOTCH1 was suggested as a protective factor for BAV related aortic aneurysm [10]. In our cohort, 9 nonsynonymous rare variants in NOTCH1 were identified in unrelated patients, with the detection rate of $9.4 \%$. The phenotypes of rare variant carriers were distinct from each other. Generally, the NOTCH1 variants were more common in the phenotype of valve dysfunction with normal aortic shape than the phenotype of aortopathy. Our findings support the argument that NOTCH1-dependent mechanism has a heterogeneity and low penetrance of aortic aneurysm in the BAV patient. Still, NOTCH1 plays the important role in the pathogenesis of BAV and in its complications.

According to the previous study and our data, the incidence of root aortopathy is ranged from 7.3 to $13.5 \%$ in patients with BAV [24]. This type of aortopathy has a tendency of rapid progress and high risk of adverse aortic events $[24,25]$. Since the root aortopathy presents a marfanoid-like aortic root morphology, it is reasonable to suggest that the genetic factors may determine a defect of the aortic wall, leading to the aortic root dilation. In our study, the pathogenic variants in TGFBR1, TGFBR2, $F L N A$, and $F B N 1$ are responsible for the aortic root dilatation. Indeed, it is a compelling finding that the pathogenic variants accounted for $57.1 \%$ of root phenotype in this consecutive BAV cohort.

The FBN1 variant has been associated with syndromic or no syndromic bicuspid aortic aneurysm [21, 26]. Meanwhile, the FLNA defects have been demonstrated to cause various developmental malformations involving the brain, skin, bone, and cardiovascular. According to Chen et al. [27], the BAV is one of the most common cardiac malformations occurred in $5.3 \%$ of the filaminopathy patients. In our group, the majority of root group patients are lack of typical systemically connective tissue disease presentations. The explanation may be the reduced penetrance of the genetic variants.

Since the phenotypes are overlapped and with low penetrance of some variants, it is challenging to make differential diagnoses with BAV related aortopathy such as fibrillinopathy and filaminopathy by only clinical manifestation. The insight of BAV genetic background helps to improve the understanding of the pathology of these diseases. In view of the fact that rare variants in some special genes act as potential modulator of BAV and associated aortopathy, the molecular diagnosis can refine the accuracy of clinical diagnosis, and thereby provides optimal guidance on patient's management and helps to develop more tailored therapeutic strategies.

\section{Limitation}

Firstly, our participants were all recruited from the surgical department. There would be a potential bias of assessing genetic effect to represent the general population. Secondly, our study method focused on the impacts of the rare variants of known BAV genes 
on the disease. Whole-genome sequencing in a larger cohort might be helpful to broaden genetic horizon of BAV and relative complications. The variants would also need further functional studies to evaluate the relevance. Long-term follow-up is also warranted for assessing the potential pathogenicity of the rare genetic variants.

\section{Conclusions}

In conclusion, this targeted NGS approach revealed a wide and specific gene variant spectrum in a large cohort of patients with BAV. From the insight of the genetic view, it is reasonable to classified the aortopathy as tubular and root type. The root phenotype presented high pathogenic variant burden among BAV patients. Patients with rare nonsynonymous coding variants had an accelerated disease progression and underwent surgical management at an early age. The molecular evaluation of BAV disease could help to accurate the diagnosis and was worth further researched.

\section{Abbreviations}

ACMG: The American College of Medical Genetics and Genomics; BAV: Bicuspid aortic valve; CNVs: Copy number variants; ExAC: The Exome aggregation consortium; NGS: Next generation sequencing.

\section{Supplementary Information}

The online version contains supplementary material available at https://doi. org/10.1186/s12872-021-02215-y.

Additional file 1: Table S1. Rare variants identified in BAV patients.

\section{Acknowledgements}

None

\section{Authors' contributions}

Mingjia Ma wrote the manuscript. Zongzhe Li sequenced the DNA samples, Mohamed Abdulkadir Mohamed collected the data. Ligang Liu carried out the interpretation and Xiang Wei designed the study. All authors read and approved the final manuscript.

\section{Funding}

This work was funded by National Natural Science Foundation of China project (No. 82070488) and Research Fund of Tongji hospital project (No. 2021A04).

\section{Availability of data and materials}

The dataset supporting the conclusions of this article is available in the NCBI Sequence Read Archive (SRA) repository, https://dataview.ncbi.n/m.nih.gov/ object/PRJNA724868? reviewer $=$ f85ctpn3hrjhi1jgmbr3dthip8

\section{Declarations}

\section{Statement of methods}

All methods in this study were carried out in accordance with relevant guidelines and regulations.

\section{Ethics approval and consent to participate}

Informed consents were acquired from all the participants, or from a parent and/or legal guardian if participants are under 18. The ethical committee of Tongji hospital, Tongji medical college, Huazhong university of science and technology approved the study.

\section{Consent for publication}

Not applicable.

\section{Competing interests}

The authors declare that they have no competing interests.

\section{Author details}

${ }^{1}$ Division of Cardiothoracic and Vascular Surgery, Tongji Hospital, Tongji Medical College, Huazhong University of Science and Technology, 1095\# Jiefang Ave., Wuhan 430030, People's Republic of China. ${ }^{2}$ Division of Cardiology, Departments of Internal Medicine and Genetic Diagnosis Center, Tongji Hospital, Tongji Medical College, Huazhong University of Science and Technology, Wuhan, People's Republic of China. ${ }^{3}$ Hubei Key Laboratory of Genetics and Molecular Mechanisms of Cardiologic Disorders, Wuhan, People's Republic of China.

Received: 30 March 2021 Accepted: 19 August 2021

Published online: 30 August 2021

\section{References}

1. Tutar E, Ekici F, Atalay S, Nacar N. The prevalence of bicuspid aortic valve in newborns by echocardiographic screening. Am Heart J. 2005;150(3):513-5.

2. Hoffman Jl, Kaplan S. The incidence of congenital heart disease. J Am Coll Cardiol. 2002;39(12):1890-900.

3. Ward C. Clinical significance of the bicuspid aortic valve. Heart. 2000:83(1):81-5.

4. Michelena HI, Desjardins VA, Avierinos JF, Russo A, Nkomo VT, Sundt TM, Pellikka PA, Tajik AJ, Enriquez-Sarano M. Natural history of asymptomatic patients with normally functioning or minimally dysfunctional bicuspid aortic valve in the community. Circulation. 2008;117(21):2776-84.

5. Yassine NM, Shahram JT, Body SC. Pathogenic mechanisms of bicuspid aortic valve aortopathy. Front Physiol. 2017;8:687

6. Michelena HI, Khanna AD, Mahoney D, Margaryan E, Topilsky Y, Suri RM, Eidem B, Edwards WD, Sundt TM 3rd, Enriquez-Sarano M. Incidence of aortic complications in patients with bicuspid aortic valves. JAMA. 2011;306(10):1104-12.

7. Mahadevia R, Barker AJ, Schnell S, Entezari P, Kansal P, Fedak PW, Malaisrie SC, McCarthy P, Collins J, Carr J, et al. Bicuspid aortic cusp fusion morphology alters aortic three-dimensional outflow patterns, wall shear stress, and expression of aortopathy. Circulation. 2014;129(6):673-82.

8. Andreassi MG, Della Corte A. Genetics of bicuspid aortic valve aortopathy. Curr Opin Cardiol. 2016;31(6):585-92.

9. Tang Y, Urs S, Boucher J, Bernaiche T, Venkatesh D, Spicer DB, Vary CP, Liaw L. Notch and transforming growth factor-beta (TGFbeta) signaling pathways cooperatively regulate vascular smooth muscle cell differentiation. J Biol Chem. 2010;285(23):17556-63.

10. Gillis E, Kumar AA, Luyckx I, Preuss C, Cannaerts E, van de Beek G, Wieschendorf B, Alaerts M, Bolar N, Vandeweyer G, et al. Candidate gene resequencing in a large bicuspid aortic valve-associated thoracic aortic aneurysm cohort: SMAD6 as an important contributor. Front Physiol. 2017;8:400

11. Sievers HH, Stierle U, Hachmann RM, Charitos El. New insights in the association between bicuspid aortic valve phenotype, aortic configuration and valve haemodynamics. Eur J Cardio-thoracic Surg Off J Eur Assoc Cardio-thoracic Surg. 2016;49(2):439-46.

12. Erbel R, Aboyans V, Boileau C, Bossone E, Bartolomeo RD, Eggebrecht $H$, Evangelista A, Falk V, Frank H, Gaemperli O et al: 2014 ESC Guidelines on the diagnosis and treatment of aortic diseases: Document covering acute and chronic aortic diseases of the thoracic and abdominal aorta of the adult. The Task Force for the Diagnosis and Treatment of Aortic Diseases of the European Society of Cardiology (ESC). European heart journal 2014, 35(41):2873-2926. 
13. http://www.internationalgenome.org/.

14. http://gnomad.broadinstitute.org.

15. http://genetics.bwh.harvard.edu/pph2/.

16. http://cadd.gs.washington.edu/.

17. Bonachea EM, Zender G, White P, Corsmeier D, Newsom D, Fitzgerald-Butt S, Garg V, McBride KL. Use of a targeted, combinatorial next-generation sequencing approach for the study of bicuspid aortic valve. BMC Med Genomics. 2014;7:56.

18. Dargis N, Lamontagne M, Gaudreault N, Sbarra L, Henry C, Pibarot P, Mathieu P, Bosse Y. Identification of gender-specific genetic variants in patients with bicuspid aortic valve. Am J Cardiol. 2016;117(3):420-6.

19. Girdauskas E, Geist L, Disha K, Kazakbaev I, Gross T, Schulz S, Ungelenk M, Kuntze T, Reichenspurner H, Kurth I. Genetic abnormalities in bicuspid aortic valve root phenotype: preliminary results. Eur J Cardio-thoracic Surg Off J Eur Assoc Cardio-thoracic Surg. 2017;52(1):156-62.

20. Prakash S, Kuang SQ, Gen TACRI, Regalado E, Guo D, Milewicz D: Recurrent rare genomic copy number variants and bicuspid aortic valve are enriched in early onset thoracic aortic aneurysms and dissections. PloS One 2016;11(4):e0153543

21. Pepe G, Nistri S, Giusti B, Sticchi E, Attanasio M, Porciani C, Abbate R, Bonow RO, Yacoub M, Gensini GF. Identification of fibrillin 1 gene mutations in patients with bicuspid aortic valve (BAV) without Marfan syndrome. BMC Med Genet. 2014;15:23.

22. Riley MF, McBride KL, Cole SE. NOTCH1 missense alleles associated with left ventricular outflow tract defects exhibit impaired receptor processing and defective EMT. Biochem Biophys Acta. 2011;1812(1):121-9.
23. Kent KC, Crenshaw ML, Goh DL, Dietz HC. Genotype-phenotype correlation in patients with bicuspid aortic valve and aneurysm. J Thorac Cardiovasc Surg. 2013;146(1):158-165 e151.

24. Della Corte A, Bancone C, Buonocore M, Dialetto G, Covino FE, Manduca S, Scognamiglio G, D'Oria V, De Feo M. Pattern of ascending aortic dimensions predicts the growth rate of the aorta in patients with bicuspid aortic valve. JACC Cardiovasc Imaging. 2013;6(12):1301-10.

25. Girdauskas E, Disha K, Rouman M, Espinoza A, Borger MA, Kuntze T. Aortic events after isolated aortic valve replacement for bicuspid aortic valve root phenotype: echocardiographic follow-up study. Eur J Cardio-thoracic Surg Off J Eur Assoc Cardio-thoracic Surg. 2015;48(4):e71-76.

26. Nistri S, Porciani MC, Attanasio M, Abbate R, Gensini GF, Pepe G. Association of Marfan syndrome and bicuspid aortic valve: frequency and outcome. Int J Cardiol. 2012;155(2):324-5.

27. Chen MH, Choudhury S, Hirata M, Khalsa S, Chang B, Walsh CA. Thoracic aortic aneurysm in patients with loss of function Filamin A mutations: clinical characterization, genetics, and recommendations. Am J Med Genet A. 2018;176(2):337-50.

\section{Publisher's Note}

Springer Nature remains neutral with regard to jurisdictional claims in published maps and institutional affiliations.
Ready to submit your research? Choose BMC and benefit from:

- fast, convenient online submission

- thorough peer review by experienced researchers in your field

- rapid publication on acceptance

- support for research data, including large and complex data types

- gold Open Access which fosters wider collaboration and increased citations

- maximum visibility for your research: over $100 \mathrm{M}$ website views per year

At BMC, research is always in progress.

Learn more biomedcentral.com/submissions 